\title{
FINITE TYCZ EXPANSIONS AND CSCK METRICS
}

\author{
ANDREA LOI, ROBERTO MOSSA, AND FABIO ZUDDAS
}

\begin{abstract}
Let $(M, g)$ be a Kähler manifold whose associated Kähler form $\omega$ is integral and let $(L, h) \rightarrow(M, \omega)$ be a quantization hermitian line bundle. In this paper we study those Kähler manifolds $(M, g)$ admitting a finite TYCZ expansion, namely those for which the associated Kempf distortion function $T_{m g}$ is of the form:

$T_{m g}(p)=f_{s}(p) m^{s}+f_{s-1}(p) m^{s-1}+\cdots+f_{r}(p) m^{r}, \quad f_{j} \in \mathcal{C}^{\infty}(M), s, r \in \mathbb{Z}$.

We show that if the TYCZ expansion is finite then $T_{m g}$ is indeed a polynomial in $m$ of degree $n, n=\operatorname{dim}_{\mathbb{C}} M$, and the log-term of the Szegö kernel of the disc bundle $D \subset L^{*}$ vanishes (where $L^{*}$ is the dual bundle of $L$ ). Moreover, we provide a complete classification of the Kähler manifolds admitting finite TYCZ expansion either when $M$ is a complex curve or when $M$ is a complex surface with a cscK metric which admits a radial Kähler potential.
\end{abstract}

\section{Contents}

1. Introduction

2. The proof of Theorem 1.1

3. Radial projectively induced cscK metrics with $a_{3}=0 \quad 12$

4. The proofs of Theorem 1.2 and Theorem $1.3 \quad 19$

References

\section{INTRODUCTION}

Let $M$ be a (not necessarily compact) $n$-dimensional complex manifold endowed with a Kähler metric $g$. Assume that there exists a holomorphic line bundle $L$ over $M$ such that $c_{1}(L)=[\omega]$, where $\omega$ is the Kähler form associated to $g$ and $c_{1}(L)$ denotes the first Chern class of $L$ (such an $L$ exists if and only if $\omega$ is an integral

2000 Mathematics Subject Classification. 53D05; 53C55; 53D05; 53D45.

Key words and phrases. TYCZ expansion; Szegö kernel; log-term; Kempf distortion function; radial potential; constant scalar curvature metric.

The first two authors were supported by Prin 2015 - Real and Complex Manifolds; Geometry, Topology and Harmonic Analysis - Italy, by INdAM. GNSAGA - Gruppo Nazionale per le Strutture Algebriche, Geometriche e le loro Applicazioni, by GESTA - Funded by Fondazione di Sardegna and Regione Autonoma della Sardegna and by KASBA- Funded by Regione Autonoma della Sardegna. 
form). Let $m \geq 1$ be an integer and let $h_{m}$ be an Hermitian metric on $L^{m}=L^{\otimes m}$ such that its Ricci curvature $\operatorname{Ric}\left(h_{m}\right)=m \omega$. Here $\operatorname{Ric}\left(h_{m}\right)$ is the two-form on $M$ whose local expression is given by

$$
\operatorname{Ric}\left(h_{m}\right)=-\frac{i}{2 \pi} \partial \bar{\partial} \log h_{m}(\sigma(x), \sigma(x)),
$$

for a trivializing holomorphic section $\sigma: U \rightarrow L^{m} \backslash\{0\}$. In the quantum mechanics terminology $L^{m}$ is called the prequantum line bundle, the pair $\left(L^{m}, h_{m}\right)$ is called a geometric quantization of the Kähler manifold $(M, m \omega)$ and $\hbar=m^{-1}$ plays the role of Planck's constant (see e.g. 1]). Consider the separable complex Hilbert space $\mathcal{H}_{m}$ consisting of global holomorphic sections $s$ of $L^{m}$ such that

$$
\langle s, s\rangle_{m}=\int_{M} h_{m}(s(p), s(p)) \frac{\omega^{n}}{n !}<\infty .
$$

Define the Kempf distortion function 1 , namely the smooth function on $M$ defined by:

$$
T_{m g}(p)=\sum_{j=0}^{N(m)} h_{m}\left(s_{j}(p), s_{j}(p)\right),
$$

where $s_{j}, j=0, \ldots, N(m)\left(\operatorname{dim} \mathcal{H}_{m}=N(m)+1 \leq \infty\right)$ is an orthonormal basis of $\mathcal{H}_{m}$.

As suggested by the notation this function depends only on the metric $m g$ and not on the orthonormal basis chosen. Obviously if $M$ is compact $\mathcal{H}_{m}=H^{0}\left(L^{m}\right)$, where $H^{0}\left(L^{m}\right)$ is the (finite dimensional) space of global holomorphic sections of $L^{m}$.

By applying the methods developed in [5] and specifically the parametrix for the Szegö kernel, D. Catlin [10] and S. Zelditch [35] independently proved that if in the above setting $M$ is compact, there exists a complete asymptotic expansion of the Kempf distortion function:

$$
T_{m g}(p) \sim \sum_{j=0}^{\infty} a_{j}(p) m^{n-j},
$$

where $a_{0}(p)=1$ and $a_{j}(p), j=1, \ldots$ are smooth functions on $M$. This means that, for any nonnegative integers $r, k$ the following estimate holds:

$$
\left\|T_{m g}(p)-\sum_{j=0}^{k} a_{j}(p) m^{n-j}\right\|_{C^{r}} \leq C_{k, r} m^{n-k-1},
$$

\footnotetext{
${ }^{1}$ In the literature the function $T_{m g}$ was first introduced under the name of $\eta$-function by $\mathrm{J}$. Rawnsley in [31, later renamed as $\theta$-function in 9 , followed by the distortion function of G. R. Kempf [16] and S. Ji [17, for the special case of Abelian varieties and of S. Zhang [34 for complex projective varieties.
} 
where $C_{k, r}$ are constant depending on $k, r$ and on the Kähler form $\omega$ and $\|\cdot\|_{C^{r}}$ denotes the $C^{r}$ norm. The expansion (3) is called Tian-Yau-Catlin-Zelditch expansion (TYCZ expansion in the sequel). Later on, Z. Lu [27], by means of Tian's peak section method, proved that each of the coefficients $a_{j}(p)$ is a polynomial of the curvature and its covariant derivatives at $p$ of the metric $g$ which can be found by finitely many algebraic operations. In particular, he computed the first three coefficients. The first two are given by:

$$
\left\{\begin{array}{l}
a_{1}(p)=\frac{1}{2} \operatorname{scal}_{g} \\
a_{2}(p)=\frac{1}{3} \Delta \text { scal }_{g}+\frac{1}{24}\left(|R|^{2}-4 \mid \operatorname{Ric}^{2}+3 \text { scal }_{g}^{2}\right)
\end{array}\right.
$$

where scal $g$, Ric, $R$, are, respectively, the scalar curvature, the Ricci tensor and the Riemann curvature tensor of $(M, g)$, in local coordinates. The reader is also referred to [18 and [19] for a recursive formula for the coefficients $a_{j}$ 's and an alternative computation of $a_{j}$ for $j \leq 3$ using Calabi's diastasis function (see also [25] for the case of locally Hermitian symmetric spaces). When $M$ is noncompact, there is not a general theorem which assures the existence of an asymptotic expansion (3). Observe that in this case we say that an asymptotic expansion (3) exists if (4) holds for any compact subset of M. M. Engliš [13] showed that a TYCZ expansion exists in the case of strongly pseudoconvex bounded domains in $\mathbb{C}^{n}$ with real analytic boundary, and proved that the first three coefficients are the same as those computed by $\mathrm{Lu}$ for compact manifolds. The reader is referred to [29] for the description of some curvature conditions which assure the existence of a TYCZ expansion in the noncompact case (see also [15] and 24] for some explicit examples).

Consider the negative Hermitian line bundle $\left(L^{*}, h^{*}\right)$ over $(M, g)$ dual to $(L, h)$ and let $D \subset L^{*}$ be the unit disk bundle over $M$, i.e.

$$
D=\left\{v \in L^{*} \mid \rho(v):=1-h^{*}(v, v)>0\right\} .
$$

It is not hard to see (and well-known) that the condition $\operatorname{Ric}(h)=\omega$ implies that $D$ is a strongly pseudoconvex domain in $L^{*}$ with smooth boundary $X=\partial D=\{v \in$ $\left.L^{*} \mid \rho(v)=0\right\}$. X will be called the unit circle bundle. Let $\mathcal{S}(v)$ be the Szegö kernel of $D$ (see Section 2 below). By a fundamental result due to Boutet de Monvel and Sjöstrand [5] 2 there exist $a, b \in C^{\infty}(\bar{D}), a \neq 0$ on $X$ such that:

$$
\mathcal{S}(v)=a(v) \rho(v)^{-n-1}+b(v) \log \rho(v), v \in D .
$$

The function $b(v) \log \rho(v)$ in (7) is called the logarithmic term (log-term from now on) of the Szegö kernel. One says that the log-term of the Szegö kernel of the disk bundle $D \subset L^{*}$ vanishes if $b=0$ identically on $D$. The Szegö kernel is strictly

${ }^{2}$ This formula (7) has been proved for strictly pseudoconvex complex domains in $\mathbb{C}^{n}$ with smooth boundary, but it could be easily extended to the disc bundle $D \subset L^{*}$ (see, e.g., 28]). 
related to the Kempf distortion function. Indeed Z. Lu and G. Tian [28] prove that 3 if the log-term of the disk bundle $D \subset L^{*}$ vanishes then $a_{k}=0$ for $k>n$, where $a_{k}$ are the coefficients appearing in (3). A conjecture still open, due to a private communication with $\mathrm{Z}$. Lu, asks if the vanishing of the $a_{k}$ 's for $k>n$ implies the vanishing of the log-term.

In this paper we address the problem of studying those Kähler manifolds whose TYCZ expansion is finite, namely the Kempf distortion function is of the form:

$$
T_{m g}(p)=f_{s}(p) m^{s}+f_{s-1}(p) m^{s-1}+\cdots+f_{r}(p) m^{r}, \quad f_{j} \in \mathcal{C}^{\infty}(M), s, r \in \mathbb{Z} .
$$

Notice that this sort of problem has been partially investigated in the compact setting by the first author of the present paper and by C. Arezzo [1].

One can give a quantum-geometric interpretation of $T_{m g}$ as follows. Assume that there exists $m$ sufficiently large such that for each point $x \in M$ there exists $s \in \mathcal{H}_{m}$ non-vanishing at $x$ (such an $m$ exists if $M$ is compact by standard algebraic geometry methods and corresponds to the free-based point condition in Kodaira's theory). Consider the so called coherent states map, namely the holomorphic map of $M$ into the complex projective space $\mathbb{C} P^{N(m)}$ given by:

$$
\varphi_{m}: M \rightarrow \mathbb{C} P^{N(m)}: x \mapsto\left[s_{0}(x): \cdots: s_{N(m)}(x)\right] .
$$

One can prove (see, e.g. 2]) that

$$
\varphi_{m}^{*} \omega_{F S}=m \omega_{g}+\frac{i}{2 \pi} \partial \bar{\partial} \log T_{m g},
$$

where $\omega_{F S}$ is the Fubini-Study form on $\mathbb{C} P^{N(m)}$, namely the Kähler form which in homogeneous coordinates $\left[Z_{0}, \ldots, Z_{N(m)}\right]$ reads as $\omega_{F S}=\frac{i}{2 \pi} \partial \bar{\partial} \log \sum_{j=0}^{N(m)}\left|Z_{j}\right|^{2}$. Recall that a Kähler metric $g$ on a complex manifold $M$ is said to be projectively induced if there exists a Kähler (isometric and holomorphic) immersion of $(M, g)$ into the finite or infinite dimensional complex projective space $\left(\mathbb{C} P^{N}, g_{F S}\right), N \leq+\infty$, endowed with the Fubini-Study metric $g_{F S}$. The reader is referred to [26] for further details and for un updated account on projectively induced Kähler metrics. Obviously not all Kähler metrics are projectively induced. Nevertheless, by combining (10) and the existence of a TYCZ expansion one gets that $\frac{\varphi_{m}^{*} g_{F S}}{m} C^{\infty}$-converges to $g$. In other words, any metric $g$ with integral Kähler form $\omega$ on a complex manifold is the $C^{\infty}$-limit of (normalized) projectively induced Kähler metrics (under the assumption of the existence of a TYCZ expansion). In the compact case this was a conjecture of Yau proved by G. Tian [33] and W. D. Ruan 32] by means of peak section method.

The following theorem represents our first result.

\footnotetext{
${ }^{3}$ The proof is given in the compact setting but it is of local nature so it immediately extends to the noncompact one.
} 
Theorem 1.1. Let $(M, g)$ be a Kähler manifold with integral Kähler form $\omega$ and of complex dimension n. Assume that the corresponding TYCZ expansion is finite. Then $T_{m g}(p)$ is forced to be a polynomial in $m$ of degree $n$ and the log-term of the Szegö kernel of the disc bundle D vanishes.

The concept of finite TYCZ expansion is strictly related to regular quantizations introduced in [9] in the context of the quantization by deformation of Kähler manifolds. One says that the quantization $(L, h)$ of a Kähler manifold $(M, g)$ is regular if the Kempf distortion function $T_{m g}$ (exists and) is a strictly positive constant for all $m$ sufficiently large (see also [20] and $[\underline{8}$ and reference therein). In S. Donaldson [11] terminology a Kähler metric $g$ with integral Kähler form $\omega$ such that its Kempf distortion function is a positive constant is called balanced. Hence a quantization of a Kähler manifold $(M, g)$ is regular if $m g$ is balanced for all $m$ sufficiently large.

When $(M, g)$ is a compact Kähler manifold which admits a regular quantization then the TYCZ expansion is necessarily finite. Indeed in that case $T_{m g}=\frac{h^{0}\left(L^{m}\right)}{V(M)}$, where $h^{0}\left(L^{m}\right)$ denotes the complex dimension of $H^{0}\left(L^{m}\right)$ and $V(M)=\int_{M} \frac{\omega^{n}}{n !}$ is the volume of $M$, and so by Riemann-Roch theorem $T_{m g}$ is a monic polynomial in $m$ of degree $n$. Thus, the vanishing of the log-term of the Szegö kernel in the last part of Theorem 1.1 (which is in accordance with the above mentioned Lu's conjecture) extends the results obtained in [4] and 21] in the compact and regular case. We believe that in the compact case, finite TYCZ expansion implies regular quantization.

Nevertheless, in the noncompact case there exist Kähler manifolds with nonconstant Kempf distortion function and finite TYCZ expansion. In order to describe an example assume that $M$ is a complex domain (open and connected) of $\mathbb{C}^{n}$ equipped with a global Kähler potential $\Phi: M \rightarrow \mathbb{R}$, i.e. $\omega=\frac{i}{2 \pi} \partial \bar{\partial} \Phi$. In this case $\omega$ is trivially integral and the Hilbert space $\mathcal{H}_{m}$ agrees with $\mathcal{H}_{m \Phi}$ the weighted Hilbert space of square integrable holomorphic functions on $M$, with weight $e^{-m \Phi}$, namely

$$
\mathcal{H}_{m \Phi}=\left\{\left.f \in \operatorname{Hol}(M)\left|\int_{M} e^{-m \Phi}\right| f\right|^{2} \frac{\omega^{n}}{n !}<\infty\right\} .
$$

If $\mathcal{H}_{m \Phi} \neq\{0\}$ then the Kempf distortion function reads as

$$
T_{m g}(z)=e^{-m \Phi(z)} K_{m \Phi}(z, z),
$$

where $K_{m \Phi}(z, z)=\sum_{j=0}^{N(m)}\left|f_{j}(z)\right|^{2}$ is the weighted reproducing kernel and $\left\{f_{j}\right\}$ an orthonormal basis for $\mathcal{H}_{m \Phi}$. Let now $p$ be a positive real number and

$$
M=\left\{\left.\left(z_{1}, z_{2}\right) \in \mathbb{C}^{2}|| z_{1}\right|^{2}+\left|z_{2}\right|^{\frac{2}{p}}<1\right\}
$$

equipped with the Kähler form $\omega$, with Kähler potential

$$
\Phi=-\log \left[\left(1-\left|z_{1}\right|^{2}\right)^{p}-\left|z_{2}\right|^{2}\right] .
$$


A straightforward computation (see e.g. [12, pp. 450-451]) shows that the weighted reproducing kernel is given by

$$
K_{m \Phi}(z, z)=e^{m \Phi}\left[m^{2}+(c(z)-3) m+c(z)+2\right]
$$

where

$$
c(z)=\left(1-\frac{1}{p}\right)\left(1-\frac{\left|z_{2}\right|^{2}}{\left(1-\left|z_{1}\right|^{2}\right)^{p}}\right) .
$$

Thus, by (12), the Kempf distortion function reads as

$$
T_{m g}(z)=m^{2}+(c(z)-3) m+c(z)+2 .
$$

It follows that for $p \neq 1, T_{m g}$ is a polynomial in $m$ of degree 2 with nonconstant coefficients $\left(a_{1}(z)=c(z)-3\right.$ and $\left.a_{2}(z)=c(z)+2\right)$. Notice that for $p=1, M$ is the complex hyperbolic plane, namely the unit ball in $\mathbb{C}^{2}$ and $\omega$ equals the hyperbolic form, and in this case the quantization is regular (see also below).

Our second result shows that, for a complex curve, finite TYCZ expansion implies regular quantization and that this happens only in the complex space form case.

Theorem 1.2. Let $M$ be a complex curve which admits a complete Kähler metric $g$ whose corresponding TYCZ expansion is finite. Then $(M, g)$ is Kähler equivalent to one of the following complex space forms:

(a) $\left(\mathbb{C}, g_{0}\right)$, where $g_{0}$ is the flat metric on $\mathbb{C}$.

(b) $\left(\mathbb{C H}^{1}, \mu g_{\text {hyp }}\right)$, where $g_{\text {hyp }}$ is the hyperbolic metric on the unit disk of $\mathbb{C}$ and $\mu$ is a positive real number.

(c) $\left(\mathbb{C P}^{1}, \lambda g_{F S}\right)$, where $g_{F S}$ is the Fubini-Study metric and $\lambda$ is a positive integer.

Many examples of Kähler manifolds admitting regular quantizations are obtained by taking simply-connected homogeneous Kähler manifolds with integral Kähler forms (see 2]). Hence, for example the complex space forms namely the flat space $\left(\mathbb{C}^{n}, g_{0}\right)$ with the flat Kähler form $\omega_{0}=\frac{i}{2 \pi} \partial \bar{\partial}|z|^{2}$, the hyperbolic space $\left(\mathbb{C H}^{n}, g_{\text {hyp }}\right)$, i.e. the unit ball in $\mathbb{C}^{n}$ with the hyperbolic form $\omega_{\text {hyp }}=-\frac{i}{2 \pi} \partial \bar{\partial} \log \left(1-|z|^{2}\right)$, the complex projective space $\left(\mathbb{C P}^{n}, g_{F S}\right)$, admit regular quantizations which, as one can easily verify, have finite TYCZ expansion.

While in the compact case the homogeneous Kähler manifolds are the only known examples admitting a regular quantization, in the noncompact case the first author together with F. C. Aghedu [8] prove that the Kempf distortion function for the Simanca metric $g_{S}$ on the blow-up $\tilde{\mathbb{C}}^{2}$ of $\mathbb{C}^{2}$ at the origin is given by $T_{m g_{S}}=m^{2}$. Hence, for the Simanca metric the quantization is not just regular but the TYCZ is finite with constant coefficients (all the coeffcients $a_{k}=0$ for $k \geq 1$ ). Notice that, if $H$ denotes the exceptional divisor, then $g_{S}$ has radial Kähler potential on 
the dense subset $U=\tilde{\mathbb{C}}^{2} \backslash H=\mathbb{C}^{2} \backslash\{0\}$ given by

$$
\Phi(z)=\left|z_{1}\right|^{2}+\left|z_{2}\right|^{2}+\log \left(\left|z_{1}\right|^{2}+\left|z_{2}\right|^{2}\right) .
$$

Our third and last result shows that the complete complex surfaces with a cscK (Kähler with constant scalar curvature) metric with densily defined radial potential and finite TYCZ expansion are essentially the complex space forms and $\left(\tilde{\mathbb{C}}^{2}, g_{S}\right)$.

Theorem 1.3. Let $M$ be a complex surface which admits a complete cscK metric $g$ whose corresponding TYCZ expansion is finite. Assume, moreover, that the metric $g$ admits a radial Kähler potential $\Phi: U \rightarrow \mathbb{R}$ defined on a dense subset $U$ of $M$. Then $(M, g)$ is Kähler equivalent to one of the following Kähler surfaces:

(i) $\left(\mathbb{C}^{2}, g_{0}\right)$, where $g_{0}$ is the flat metric on $\mathbb{C}^{2}$.

(ii) $\left(\mathbb{C H}^{2}, \mu g_{h y p}\right)$, where $g_{\text {hyp }}$ is the hyperbolic metric on the unit disk of $\mathbb{C}^{2}$ and $\mu$ is a positive real number.

(iii) $\left(\mathbb{C P}^{2}, \lambda g_{F S}\right)$, where $g_{F S}$ is the Fubini-Study metric and $\lambda$ is a positive integer.

(iv) $\left(\tilde{\mathbb{C}}^{2}, \lambda g_{S}\right)$, where $\tilde{\mathbb{C}}^{2}$ denotes the blow-up of $\mathbb{C}^{2}$ at the origin, $g_{S}$ the Simanca metric and $\lambda$ is a positive integer.

Remark 1. The assumption on the potential in Theorem 1.3 means that $U$ can be equipped with global complex coordinates $z_{1}$ and $z_{2}$ and $\Phi$ only depends on $\left|z_{1}\right|^{2}+\left|z_{2}\right|^{2}$. Notice also that $\Phi$ is not necessarily defined at the origin (see Remark 4 below for details).

Remark 2. If we assume $M=\mathbb{C P}^{2}$ and the finiteness of TYCZ expansion then, by using the last part of Theorem 1.1, one can get that $g=\lambda g_{F S}$ for some integer $\lambda$, without further assumptions (either on the curvature or on the potential). Indeed, a deep result due to Z. Lu and G. Tian [28] asserts that an integral Kähler form on $\mathbb{C P}^{2}$ such that the log-term of the disk bundle vanishes is an integral positive mutiple of the Fubini-Study form.

The paper is organized as follows. Section 2 and Section 4 are dedicated to the proofs of Theorem 1.1 and Theorems 1.2 1.3 respectively. The proof of the latter is based on the classification of radial cscK projectively induced metrics with $a_{3}=0$ given in Section 3 (see Proposition 3.1).

\section{The PRoOf of TheOREM 1.1}

Let $(M, g)$ be a Kähler manifold. Assume that the Kähler form $\omega$ associated to $g$ is integral and let $(L, h) \rightarrow(M, \omega)$ be a quantization bundle and $D \subset L^{*}$ be the corresponding disk bundle as in the introduction. The proof of Theorem 1.1 is based on the link between the Szegö kernel of the disk bundle $D$ and the Kempf 
distortion function (see Equation (20) below) and on the two subsequent lemmata (Lemma 2.1 and Lemma 2.2). In order to obtain (20) let us denote by $\mathcal{H}^{2}(X)$ the space of boundary values of holomorphic functions on $D$ that are square integrable on $X$ with respect to the measure $d \mu=\theta \wedge(d \theta)^{n}$, being $d \theta=\omega$. The Hardy space $\mathcal{H}^{2}(X)$ admits the Fourier decomposition into irreducible factors with respect to the natural $S^{1}$-action. Namely,

$$
\mathcal{H}^{2}(X)=\bigoplus_{m=0}^{+\infty} \mathcal{H}_{m}^{2}(X)
$$

where

$$
\mathcal{H}_{m}^{2}(X)=\left\{f \in \mathcal{H}^{2}(X) \mid f\left(e^{i \theta} x\right)=e^{i m \theta} f(x)\right\},
$$

equivalently, $f(\alpha v)=\alpha^{m} f(v)$ for $\alpha \in \mathbb{C}$ (since $f$ is holomorphic).

By definition the Szego kernel $\mathcal{S}(z, w)$ is the reproducing kernel of $\mathcal{H}^{2}(X)$, i.e. is characterized by the properties $\mathcal{S}(z, w) \in \mathcal{H}^{2}(X)$ for every fixed $w \in D, \mathcal{S}(w, z)=$ $\overline{\mathcal{S}(z, w)}$ and

$$
f(z)=\int_{X} \mathcal{S}(z, w) f(w) d \mu_{w}
$$

for every $f \in \mathcal{H}^{2}(X)$ and $z \in D$. From these properties it is immediately seen that $\mathcal{S}(z, w)=\sum_{j=1}^{\infty} f_{j}(z) \overline{f_{j}(w)}$, where $\left\{f_{j}\right\}$ is an orthonormal basis of $\mathcal{H}^{2}(X)$. Let us denote $\mathcal{S}(z):=\mathcal{S}(z, z)=\sum_{j=1}^{\infty}\left|f_{j}(z)\right|^{2}$. Now, by (15), an orthonormal basis of $\mathcal{H}^{2}(X)$ can be obtained by putting together orthonormal bases of $\mathcal{H}_{m}^{2}(X)$ for $m=0,1, \ldots$ If $f_{1}, \ldots, f_{N(m)}$ form an orthonormal basis of $\mathcal{H}_{m}^{2}(X)$, let us denote

$$
\mathcal{S}_{m}(v):=\sum_{j=1}^{N(m)}\left|f_{j}(v)\right|^{2}
$$

Then, we can write

$$
\mathcal{S}(v)=\sum_{m=0}^{\infty} \mathcal{S}_{m}(v)
$$

Remark 3. Notice that $\mathcal{H}_{0}^{2}(X)$ is the space of holomorphic functions $f$ on $D$ such that $f\left(e^{i \theta} x\right)=f(x)$ for every $x \in X$, i.e. the functions which are constant on the fiber above every point $p \in M$ and square integrable on $X$. If $M$ is compact, $\mathcal{H}_{0}^{2}(X)$ obviously contains only the constant functions and a basis is given by $f \equiv c$ such that $\int_{X}|c|^{2} d \mu=1$. If $M$ is not compact, $\mathcal{H}_{0}^{2}(X)$ identifies with the space of holomorphic functions $f$ on $M$ such that $\int_{M}|f|^{2} \omega^{n}<\infty$, and $\mathcal{S}_{0}(v)$ is constant on each fiber of $D$, i.e. it can be identified with a smooth function $F_{0}: M \rightarrow \mathbb{C}$.

Let $\mathcal{H}_{m}$ be the space of $L^{2}$-bounded holomorphic sections of $L^{m}$ defined in the introduction. It is easy to see (see e.g. 35] for the compact case) that for $m \geq 1$ there is a unitary equivalence $\mathcal{H}_{m} \rightarrow \mathcal{H}_{m}^{2}(X)$ which sends a section $s \in \mathcal{H}_{m}$ to the 
function $\hat{s} \in \mathcal{H}_{m}^{2}(X)$ defined by

$$
\hat{s}(\lambda)=\lambda^{m}(s)
$$

for every $\lambda \in L^{*}$. Then, if we take an orthonormal basis $s_{1}, \ldots, s_{N(m)}$ of $\mathcal{H}_{m}$ then $\hat{s}_{1}, \ldots, \hat{s}_{N(m)}$ is an orthonormal basis of $\mathcal{H}_{m}^{2}(X)$.

Thus, for $m \geq 1$ we have

$$
\mathcal{S}_{m}(v)=\sum_{j=1}^{N(m)}\left|\hat{s}_{j}(v)\right|^{2}=\sum_{j=1}^{N(m)}\left|\left(\sqrt{h^{*}(v, v)}\right)^{m} \hat{s}_{j}(x)\right|^{2}=\left(h^{*}(v, v)\right)^{m} \sum_{j=1}^{N(m)}\left|\hat{s}_{j}(x)\right|^{2}
$$

where we denote by $x=\frac{v}{\sqrt{h^{*}(v, v)}}$. Thus, since $\sum_{j=1}^{N(m)}\left|\hat{s}_{j}(x)\right|^{2}$ is the Kempf distortion function $T_{m g}(\pi(x))=T_{m g}(\pi(v))$ (where $\pi: L^{*} \rightarrow M$ is the bundle projection), we have

$$
\mathcal{S}_{m}(v)=\left(h^{*}(v, v)\right)^{m} T_{m g}(\pi(v)) .
$$

Combining this with (19), we can write 4

$$
\mathcal{S}(v)=\sum_{m=0}^{\infty}\left(h^{*}(v, v)\right)^{m} T_{m g}(p)
$$

for $v \in D$, where $p=\pi(v)$ and $T_{0}(p)$ is the function $F_{0}$ in Remark 3 above.

Lemma 2.1. Let $(M, g)$ be a Kähler manifold such that Kähler form $\omega$ associated to $g$ is integral. Assume that the associated Kempf distortion function $T_{m g}$ admits a TYCZ expansion. Let $p_{0} \in M$ and define

$$
\phi(t)=\sum_{m=0}^{\infty}(1-t)^{n+1} t^{m} T_{m g}\left(p_{0}\right)
$$

Then the map $t \mapsto \phi^{(h)}(t):=\frac{\partial^{h}}{\partial t^{h}}(\phi(t))$ is bounded on $(0,1)$ for all $h \geq 0$.

Proof. One has

$$
\begin{aligned}
\phi^{(h)}(t) & =\sum_{m=0}^{\infty}\left((1-t)^{n+1} t^{m}\right)^{(h)} T_{m g}\left(p_{0}\right) \\
& =\sum_{m=1}^{\infty}\left((1-t)^{n+1} t^{m}\right)^{(h)} T_{m g}\left(p_{0}\right)+\left((1-t)^{n+1}\right)^{(h)} T_{0}\left(p_{0}\right)
\end{aligned}
$$

On the other hand, by (4), we have

$$
-C_{0,0} m^{n-1}+a_{0} m^{n} \leq T_{m g}\left(p_{0}\right) \leq C_{0,0} m^{n-1}+a_{0} m^{n}, m \geq 1 .
$$

Hence to show that $\phi^{(h)}(t)$ is bounded one needs to verify that the two functions

$$
\varphi_{k}(t)=\sum_{m=1}^{\infty}\left((1-t)^{n+1} t^{m}\right)^{(h)} m^{k}, \quad k=n-1, n
$$

\footnotetext{
${ }^{4}$ Equation (20) extends to the noncompact setting the analogous equation proved in [4] for the compact case.
} 
are bounded on $(0,1)$. This easily follows since

$$
\left(\sum_{m=0}^{\infty}(1-t)^{n+1} t^{m} m^{k}\right)^{(h)}=\left(q_{k}(t)(1-t)^{n-k}\right)^{(h)}, 0<t<1,
$$

where $q_{k}(t)$ is the polynomial of degree $k$ in $t$ such that

$$
q_{k}(t)=(1-t)^{k+1} \sum_{m=0}^{\infty} t^{m} m^{k}, 0<t<1 .
$$

Lemma 2.2. Let $k_{0}$ be a positive integer and $h$ a natural number. Consider the function

$$
\psi_{h}(t)=\left((1-t)^{n+1} \sum_{m=1}^{\infty} \frac{t^{m}}{m^{k_{0}+h}}\right)^{\left(n+k_{0}\right)} \quad, 0<t<1 .
$$

Then $\psi_{h}(t)=O(1)$ in $[0,1]$ if and only if $h \neq 0$.

Proof. Observe that

$$
\begin{aligned}
\psi_{h}(t) & =\sum_{l=k_{0}-1}^{n+k_{0}} c_{l}(1-t)^{1-k_{0}+l} \sum_{m=1}^{\infty}\left(\frac{t^{m}}{m^{k_{0}+h}}\right)^{(l)} \\
& =\sum_{l=k_{0}-1}^{n+k_{0}}(1-t)^{1-k_{0}+l} \sum_{\substack{m=l \text { if } l \geq 1 \\
m=1 \text { if } l=0}}^{\infty}\left(b_{l, l} m^{l}+b_{l, l-1} m^{l-1}+\cdots+b_{l, 1} m+b_{l, 0}\right) \frac{t^{m-l}}{m^{k_{0}+h}} \\
& =\sum_{l=k_{0}-1}^{n+k_{0}} \sum_{s=0}^{l} b_{l, s} \sum_{\substack{m=l \text { if } l \geq 1 \\
m=1 \text { if } l=0}}^{\infty}\left(\frac{t^{m-l}}{m^{k_{0}+h-s}}(1-t)^{1-k_{0}+l}\right)
\end{aligned}
$$

where $c_{l}, b_{l, l-1}, \ldots, b_{l, 0}, b_{l}$ are suitable real numbers. Consider the series

$$
F_{k_{0}, s, h, l}(t)=(1-t)^{1-k_{0}+l} \sum_{\substack{m=l \text { if } l \geq 1 \\ m=1 \text { if } l=0}}^{\infty} \frac{t^{m-l}}{m^{k_{0}+h-s}}
$$

for $k_{0}-1 \leq l \leq n+k_{0}$ and $0 \leq s \leq l$. Notice that for $l=0$ (and hence $s=0$ and $\left.k_{0}=1\right)$ (25) reads as $\sum_{m=1}^{\infty} \frac{t^{m}}{m^{h+1}}$ which is bounded for $t \rightarrow 1^{-}$if and only if $h>0$. More generally, we claim that (25) diverges if and only if $h=0$ and $s=l=k_{0}-1$.

Indeed for $h=0$ and $s=l=k_{0}-1$ (25) reads as

$$
t^{-k_{0}+1} \sum_{\substack{m=k_{0}-1 \text { if } k_{0} \geq 2 \\ m=1 \text { if } k_{0}=1}}^{\infty} \frac{t^{m}}{m}=t^{-k_{0}+1}\left[\log (1-t)-\sum_{m=1}^{k_{0}-2}\left(\frac{t^{m}}{m}\right)\right]
$$

and so it tends to $-\infty$ for $t \rightarrow 1^{-}$. On the other hand for the other values of the parameters one has the following case by case analysis which shows that (25) is bounded for $t \rightarrow 1^{-}$(we assume $l \geq 1$ by the above considerations).

Case 1. $s>k_{0}+h$ : 


$$
\begin{aligned}
F_{k_{0}, s, h, l}(t) & =(1-t)^{1-k_{0}+l} \sum_{m=l}^{\infty} \frac{t^{m-l}}{m^{k_{0}+h-s}}=(1-t)^{1-k_{0}+l} \sum_{\tilde{m}=0}^{\infty} t^{\tilde{m}}(\tilde{m}+l)^{s-k_{0}-h} \\
& =(1-t)^{l-s} \tilde{q}_{s-k_{0}-h}(t),
\end{aligned}
$$

where $\tilde{q}_{s-k_{0}-h}(t)$ is a polynomial of degree $s-k_{0}-h$.

Case 2. $s=k_{0}+h$ (and hence $l \geq k_{0}$ ):

$$
\begin{aligned}
F_{k_{0}, s, h, l}(t) & =(1-t)^{1-k_{0}+l} t^{-l} \sum_{m=l}^{\infty} t^{m}=(1-t)^{1-k_{0}+l} t^{-l}\left[\frac{1}{1-t}-\sum_{\tilde{m}=0}^{l-1} t^{\tilde{m}}\right] \\
& =(1-t)^{-k_{0}+l} t^{-l}-(1-t)^{1-k_{0}+l} t^{-l} \sum_{\tilde{m}=0}^{l-1} t^{\tilde{m}}
\end{aligned}
$$

Case 3. $s=k_{0}+h-1$ (and henct5 $l>k_{0}-1$ ):

$$
F_{k_{0}, s, h, l}(t)=(1-t)^{1-k_{0}+l} t^{-l} \sum_{m=l}^{\infty} \frac{t^{m}}{m}=(1-t)^{1-k_{0}+l} t^{-l}\left[\log (1-t)-\sum_{\tilde{m}=1}^{l-1} \frac{t^{\tilde{m}}}{\tilde{m}}\right] \text {. }
$$

Case 4. $s \leq k_{0}+h-2$ :

$$
F_{k_{0}, s, h, l}(t)=(1-t)^{1-k_{0}+l} \sum_{m=l}^{\infty} \frac{t^{m-l}}{m^{k_{0}+h-s}} \leq(1-t)^{1-k_{0}+l} \sum_{m=l}^{\infty} \frac{t^{m-l}}{m^{2}} .
$$

We can now prove Theorem 1.1 .

Proof of Theorem 1.1. We first prove that (8) forces $T_{m g}(p)$ to be a polynomial of degree $n$. By (4) for $k=0$ and (8) one gets

$$
\left|\sum_{h=r}^{s} f_{h}(p) m^{h-n}-a_{0}\right| \leq C_{0,0} m^{-1}
$$

and taking $m \rightarrow \infty$ one deduces $f_{n+1}=f_{n+2}=\ldots f_{s}=0$ and $f_{n}=a_{0}=1$. It remains to show that $r \geq 0$. Assume by a contradiction that $r<0$. Then the function $\phi(t)$ given in (21) decomposes as

$$
\phi(t)=(1-t)^{n+1} T_{0}(p)+g_{+}(t)+g_{-}(t)
$$

where

$$
\begin{gathered}
g_{+}(t):=(1-t)^{n+1} \sum_{m=1}^{\infty} t^{m}\left(f_{n}(p) m^{n}+\cdots+f_{0}(p)\right) \\
g_{-}(t):=(1-t)^{n+1} \sum_{m=1}^{\infty} t^{m}\left(f_{-1}(p) \frac{1}{m}+\cdots+f_{r}(p) \frac{1}{m^{|r|}}\right) .
\end{gathered}
$$

and there exists a positive integer $k_{0}$ such that $f_{-1}(p)=f_{-2}(p)=\cdots=f_{-k_{0}+1}(p)=$ 0 and $f_{-k_{0}}(p) \neq 0$. Notice that

$$
g_{-}^{\left(n+k_{0}\right)}(t)=\sum_{h=0}^{|r|-k_{0}} f_{-k_{0}-h}(p) \psi_{h}(t)
$$

${ }^{5}$ Since $l=k_{0}-1$ forces $h=0$ and $s=l=k_{0}-1$. 
(where $\psi_{h}(t)$ is defined by (23)) and, by Lemma 2.2.

$$
\lim _{t \rightarrow 1^{-}} g_{-}^{\left(n+k_{0}\right)}(t)=-\infty .
$$

By combining (26), (27) and the fact that $g_{+}(t)$ has bounded derivatives of all orders (being $g_{+}(t)=\sum_{k=0}^{n} f_{k}(x) q_{k}(t)(1-t)^{n-k}$, where $q_{k}(t)$ is the polynomial given by (22) $)$ we deduce that $\phi^{\left(n+k_{0}\right)}(t)$ is unbounded in contrast with Lemma 2.1

Let now $p_{0} \in M$ and $e: U \rightarrow L^{*}$ be a local trivialization on a neighborhood of $p_{0}$. Consider the coordinate system

$$
v(t, \theta, p)=\sqrt{\frac{t}{h(p)}} e^{i \theta} e(p),
$$

where $h(p)=h^{*}(e(p), e(p))$ (and hence $h^{*}(v(t, \theta, p), v(t, \theta, p))=t$ ) By (20) and $h^{*}(v(t, \theta, p), v(t, \theta, p))=t$, one has

$$
\phi(t)=\sum_{m=0}^{\infty}(1-t)^{n+1} t^{m} T_{m g}\left(p_{0}\right)=\rho(t)^{n+1} \mathcal{S}\left(v\left(t, \theta, p_{0}\right)\right)
$$

Therefore, by inserting $g_{-}(t)=0$ in (26) one obtains that $\phi: D \rightarrow \mathbb{R}$ is the restriction of a smooth function on $\bar{D}$ and by (7) one deduces that the log-term of the Szegö kernel $\mathcal{S}(v)$ of $D$ must vanish, concluding the proof of the theorem.

\section{RAdiAL PROJECTIVELY INDUCED CSCK METRICS WITH $a_{3}=0$}

We first recall the classification of radial Kähler metrics with constant scalar curvature proved in 23. Let $U \subset \mathbb{C}^{n}$ be a complex domain (not necessarily containing the origin of $\mathbb{C}^{n}$ ) endowed with a Kähler form $\omega=\frac{i}{2 \pi} \partial \bar{\partial} \Phi$ with radial potential $\Phi: U \rightarrow \mathbb{R}$, i.e.

$$
\Phi(z)=f(r), \quad r=|z|^{2}=\left|z_{1}\right|^{2}+\cdots+\left|z_{n}\right|^{2} \in \tilde{U}:=\left\{r=|z|^{2}, z \in U\right\} .
$$

These metrics can be studied by rewriting everything in terms of the function $\psi(y)$ introduced in the proof of Theorem 2.1 in 23, i.e. more precisely $F(t)=f\left(e^{t}\right)$, $y=F^{\prime}(t), \psi(y)=F^{\prime \prime}(t)$.

In particular, by assuming that $g$ is cscK, one shows after a long but straightforward calculation (see the proof of Theorem 2.1 in [23]) that $\psi$ has the form

$$
\psi(y)=A y^{2}+y+\frac{B}{y^{n-2}}+\frac{C}{y^{n-1}},
$$

where $A, B$ and $C$ are constants and the scalar curvature is equal to $-A n(n+1)$.

Remark 4. Assume $n=2$. If we set $z={ }^{t}\left(z_{1}, z_{2}\right)$ then one easily sees that the matrix of the metric $g$ (still denoted by $g$ ) reads as:

$$
g=\frac{F^{\prime \prime}-F^{\prime}}{e^{2 t}} z^{t} \bar{z}+\frac{F^{\prime}}{e^{t}} I
$$


where $I$ is the $2 \times 2$ identity matrix, whose (positive) eigenvalues are $\frac{F^{\prime}}{e^{t}}$ and $\frac{F^{\prime \prime}}{e^{t}}$. So, if we further assume that $\Phi$ is defined at the origin, we get

$$
\lim _{t \rightarrow-\infty} F^{\prime}=\lim _{t \rightarrow-\infty} F^{\prime \prime}=0,
$$

forcing $B=C=0$ in (29). In this case the solution of $\psi(y)=A y^{2}+y$ are the flat, the Fubini-Study and the hyperbolic metric if $A=0, A<0$ and $A>0$, respectively (cfr. (31), (34) and (35) below).

In the proof of Theorem 1.1 in [23] it is shown that $n=2$ and $a_{3}=0$ (where $a_{3}$ is the third coefficient of TYCZ expansion of the Kempf distortion function) if and only if $C=0$, so (29) reduces under these assumptions to

$$
\psi(y)=A y^{2}+y+B
$$

Remark 5. For a cscK radial metric the condition $a_{3}=0$ is equivalent to $a_{2}=0$ (see [23]). This fact will be used in the proof of Theorem 1.3 .

Hence the classification of radial cscK metrics with $a_{3}=0$ reduces to integrating equation (30) (recall that $\psi=y^{\prime}$, where the derivative is meant with respect to $t=\log r)$ in the cases $A=0$ and $A \neq 0$. In the latter we further distinguish the three cases where the equation $A y^{2}+y+B=0$ has no real solutions, only one real solution or two real solutions, and the sign of these solutions. Let us briefly recall the result of such classification. In order to keep the same notation used in [23, we will rewrite $\psi$ in terms of real parameters $\lambda, \mu, \xi, \zeta>0,0<\zeta<1, \kappa \in \mathbb{R}$ (the exact relation with $A, B$ is not necessary for our purposes).

When $a_{1}=0$ (namely vanishing scalar curvature or, equivalently, $A=0$ ) we have the following three cases:

$$
\psi(y)=y
$$

which corresponds to the flat metric $g_{0}$ on $U \subseteq \mathbb{C}^{2}$;

$$
\psi(y)=y-\lambda
$$

which integrates as $F^{\prime}(t)=\mu e^{t}+\lambda$, is defined on $r=e^{t}>0$ and is (a multiple of) the Simanca metric (14) on $U \subseteq \mathbb{C}^{2} \backslash\{0\}$;

$$
\psi(y)=y+\lambda
$$

which integrates as $F^{\prime}(t)=\mu e^{t}-\lambda$ and is defined on $r=e^{t}>\frac{\lambda}{\mu}$. Notice that $F^{\prime} \rightarrow 0$ when $r \rightarrow \frac{\lambda}{\mu}$.

When $a_{1} \neq 0$ (equivalently, $A \neq 0$ ) we have the following eight cases [(34)-(41)]:

$$
\psi(y)=\frac{1}{\mu} y(\mu-y)
$$


which integrates as $y=F^{\prime}(t)=\frac{\mu e^{t}}{1+e^{t}}$ and corresponds to the multiple $\mu \omega_{F S}=$ $\mu i \partial \bar{\partial} \log \left(1+|z|^{2}\right)$ of the Fubini-Study metric on $U \subseteq \mathbb{C}^{2} \subset \mathbb{C} P^{2}$;

$$
\psi(y)=\frac{1}{\mu} y(\mu+y)
$$

which integrates as $y=F^{\prime}(t)=\frac{\mu e^{t}}{1-e^{t}}$ and corresponds to the multiple $\mu \omega_{\text {hyp }}=$ $-\mu i \partial \bar{\partial} \log \left(1-|z|^{2}\right)$ of the hyperbolic metric on $U \subseteq \mathbb{C} H^{2}$;

$$
\psi(y)=\left[\left(\frac{1}{\mu} y+\frac{1}{2}\right)^{2}+\lambda^{2}\right]
$$

which is easily seen to integrate as $y=F^{\prime}(t)=\mu\left[\lambda \tan (\lambda t+\kappa)-\frac{1}{2}\right]$ with maximal interval of definition given by $h \pi+\arctan \left(\frac{1}{2 \lambda}\right)<\lambda t+\kappa<\frac{2 h+1}{2} \pi$. Notice that $F^{\prime} \rightarrow 0$ when $\lambda t+\kappa \rightarrow h \pi+\arctan \left(\frac{1}{2 \lambda}\right)$;

$$
\psi(y)=\frac{1}{\mu}\left(y-\frac{1-\zeta}{2}\right)\left(y-\frac{1-\zeta}{2}+\mu\right)
$$

which is easily seen to integrate as $y=F^{\prime}(t)=-\mu\left[\frac{-\xi \zeta e^{\zeta t}}{1-\xi e^{\zeta t}}+\frac{1-\zeta}{2}\right]$ with maximal interval of definition given by $\frac{1-\zeta}{1+\zeta}<\xi e^{\zeta t}<1$. Notice that $F^{\prime} \rightarrow 0$ when $\xi e^{\zeta t} \rightarrow$ $\frac{1-\zeta}{1+\zeta}$

$$
\psi(y)=\frac{1}{\mu}\left(y-\frac{\mu \lambda}{2}\right)\left(y+\frac{\mu \lambda}{2}+\mu\right)
$$

which is easily seen to integrate as $y=F^{\prime}(t)=-\mu\left[\frac{-\xi(\lambda+1) e^{(\lambda+1) t}}{1-\xi e^{(\lambda+1) t}}-\frac{\lambda}{2}\right]$ with maximal interval of definition given by $0<\xi e^{(\lambda+1) t}<1$. Notice that $\frac{\mu \lambda}{2}<F^{\prime}<+\infty$ :

$$
\psi(y)=-\frac{1}{(\lambda+1) \mu}\left(y+\frac{\mu \lambda}{2}\right)\left(y-\frac{\mu \lambda}{2}-\mu\right)
$$

which is easily seen to integrate as $y=F^{\prime}(t)=\mu\left[\frac{-\xi(\lambda+1) e^{-(\lambda+1) t}}{1+\xi e^{-(\lambda+1) t}}+\frac{2+\lambda}{2}\right]$ with maximal interval of definition given by $\xi e^{(\lambda+1) t}>\frac{\lambda}{2+\lambda}$. Notice that $F^{\prime} \rightarrow 0$ when $\xi e^{(\lambda+1) t} \rightarrow \frac{\lambda}{2+\lambda}$

$$
\psi(y)=-\frac{1}{\mu}\left(y-\frac{\mu(1+\zeta)}{2}\right)\left(y-\frac{\mu(1-\zeta)}{2}\right)
$$

which is easily seen to integrate as $y=F^{\prime}(t)=\mu\left[\frac{-\zeta e^{-\zeta t}}{1+e^{-\zeta t}}+\frac{1+\zeta}{2}\right]$ with maximal interval of definition $\mathbb{R} \backslash\{0\}$. Notice that $\frac{\mu(1-\zeta)}{2}<y<\frac{\mu(1+\zeta)}{2}$;

$$
\psi(y)=\frac{1}{\mu}\left(y+\frac{\mu}{2}\right)^{2}
$$

which is easily seen to integrate as $y=F^{\prime}(t)=\mu\left[\frac{1}{k-t}-\frac{1}{2}\right]$ with maximal interval of definition $k-2<t<k$. Notice that $F^{\prime} \rightarrow 0$ when $t \rightarrow k-2$.

Metrics (36)-(41) correspond respectively to cases 11a, 6, 7, 8, 9, 10a of Theorem 2.1 in 23 . 
In 23] the first and the third author together with F. Salis proved that the flat metric and the Simanca metric, i.e. the cases (31) and (32) above, are the only radial projectively induced metrics with $a_{1}=a_{3}=0$.

Remark 6. For the hyperbolic metric $g_{\text {hyp }}$ on $\mathbb{C} H^{n}$ and the Fubini-Study metric $g_{F S}$ on $\mathbb{C}^{n}$, i.e. the cases (35) and (34) above, one has that $\mu g_{\text {hyp }}$ admits an injective Kähler immersion into $\mathbb{C} P^{\infty}$ for any $\mu>0$, while $\mu g_{F S}$ admits an injective Kähler immersion into $\mathbb{C} P^{N}$ if and only if $\mu$ is an integer (the reader is referred to [6] for an explicit descriptions of these maps).

Among the other cases above, it is easy to see that the metric (38) is projectively induced provided $\lambda \in \mathbb{Z}$ and $\frac{\lambda \mu}{2} \in \mathbb{Z}$. Indeed, an explicit potential of this metric is given by

$$
\hat{\Phi}=\log \frac{\left(\left|z_{1}\right|^{2}+\left|z_{2}\right|^{2}\right)^{\frac{\mu \lambda}{2}}}{\left[1-\xi\left(\left|z_{1}\right|^{2}+\left|z_{2}\right|^{2}\right)^{(\lambda+1)}\right]^{\mu}}
$$

and, by using $\frac{1}{(1-x)^{\mu}}=\sum_{i=0}^{\infty} \frac{\mu(\mu+1) \cdots(\mu+i-1)}{i !} x^{i}$ one has

$$
\begin{aligned}
e^{\hat{\Phi}} & =\left(\left|z_{1}\right|^{2}+\left|z_{2}\right|^{2}\right)^{\frac{\mu \lambda}{2}} \sum_{i=0}^{\infty} \frac{\mu(\mu+1) \cdots(\mu+i-1)}{i !} \xi^{i}\left(\left|z_{1}\right|^{2}+\left|z_{2}\right|^{2}\right)^{(\lambda+1) i} \\
& =\sum_{i=0}^{\infty} \frac{\mu(\mu+1) \cdots(\mu+i-1)}{i !} \xi^{i}\left(\left|z_{1}\right|^{2}+\left|z_{2}\right|^{2}\right)^{(\lambda+1) i+\frac{\mu \lambda}{2}} \\
& =\sum_{i=0}^{\infty} \sum_{j=0}^{(\lambda+1) i+\frac{\mu \lambda}{2}} \frac{\mu(\mu+1) \cdots(\mu+i-1)}{i !} \xi^{i}\left(\begin{array}{c}
(\lambda+1) i+\frac{\mu \lambda}{2} \\
j
\end{array}\right)\left|z_{1}\right|^{2 j}\left|z_{2}\right|^{2(\lambda+1) i+\mu \lambda-2 j} .
\end{aligned}
$$

Then,

$$
\left(z_{1}, z_{2}\right) \mapsto\left[\cdots, \sqrt{\frac{\mu(\mu+1) \cdots(\mu+i-1)}{i !} \xi^{i}\left(\begin{array}{c}
(\lambda+1) i+\frac{\mu \lambda}{2} \\
j
\end{array}\right)} z_{1}^{j} z_{2}^{k}, \cdots\right]
$$

for $i=0, \ldots, \infty$ and $j+k=(\lambda+1) i+\frac{\mu \lambda}{2}$ gives the desired projective immersion.

Remark 7. Notice that the metric given by potential (42) is not Einstein for any values of $\lambda, \mu, \xi>0$. Indeed, for a Kähler metric $g$ on a 2-dimensional manifold with Kähler form $\omega=\frac{i}{2 \pi} \partial \bar{\partial} \Phi$ given by a radial potential $\Phi(z)=f(r), r=\left|z_{1}\right|^{2}+\left|z_{2}\right|^{2}$, we have

$$
\operatorname{det}(g)=\operatorname{det}\left(\begin{array}{cc}
f^{\prime}+f^{\prime \prime} \cdot\left|z_{1}\right|^{2} & f^{\prime \prime} \bar{z}_{1} z_{2} \\
f^{\prime \prime} \bar{z}_{2} z_{1} & f^{\prime}+f^{\prime \prime} \cdot\left|z_{2}\right|^{2}
\end{array}\right)=f^{\prime 2}+f^{\prime} f^{\prime \prime} r=f^{\prime}\left(r f^{\prime}\right)^{\prime} .
$$

Then, after a straightforward computation with $\Phi$ given by (42) we get 


$$
\operatorname{det}(g)=\frac{\xi \mu^{2}(\lambda+1)^{2}}{2} \frac{r^{\lambda-1}\left[\lambda+\xi(\lambda+2) r^{\lambda+1}\right]}{\left(1-\xi r^{\lambda+1}\right)^{3}}
$$

and one immediately sees that the metric is not Einstein by comparing $\log \operatorname{det}(g)$ with (42).

The following proposition, interesting on its own sake, shows that the only radial cscK projectively induced metrics with $a_{3}=0$ are those just described. It could be interesting to classify all the radial projectively induced cscK metrics without the assumption of the vanishing of $a_{3}$ (the reader is referred to 22 for the classification of radial projectively induced Ricci flat Kähler metrics).

Proposition 3.1. Let $U \subset \mathbb{C}^{n}$ be a complex domain on which is defined a radial Kähler metric $g$ given by a radial potential $\Phi: U \rightarrow \mathbb{R}$. Assume that $g$ is a cscK metric and $a_{3}=0$. Then $g$ is projectively induced if and only if we are in the cases (31), (32) with $\lambda \in \mathbb{Z}$, 34) with $\mu \in \mathbb{Z}$, (35) for any $\mu$ and (38) with $\lambda, \frac{\lambda \mu}{2} \in \mathbb{Z}$, of the above classification.

In order to prove the proposition we need three lemmata.

Lemma 3.2. Let $U \subset \mathbb{C}^{n}$ be a complex domain endowed with a Kähler metric $g$ whose associated Kähler form $\omega=\frac{i}{2 \pi} \partial \bar{\partial} \Phi$ hass radial potential $\Phi: U \rightarrow \mathbb{R}$, i.e. $\Phi(z)=f(r), r=|z|^{2}$. If there exist $r \in \tilde{U}:=\left\{r=|z|^{2}, z \in U\right\}$ and $h \in \mathbb{N}$ such that

$$
g_{h}(r)=\frac{d^{h} e^{f(r)}}{d r^{h}}<0
$$

then $g$ is not projectively induced.

Proof. See Lemma 3.1 in [22] for a proof.

Lemma 3.3. Let $g$ be a radial Kähler metric as above and let $\psi(y)=A y^{2}+y+B$ given by (30). Assume $g$ is projectively induced and 0 is a limit point in the domain of definition of $\psi$. Then $B=0$ (i.e. $\psi$ has 0 as root).

Proof. By taking $h=1,2,3$ in (45), one gets in particular that if $g$ is projectively induced then

$$
\begin{gathered}
f^{\prime} \geq 0 \\
f^{\prime 2}+f^{\prime \prime} \geq 0 \\
f^{\prime \prime \prime}+3 f^{\prime} f^{\prime \prime}+f^{\prime 3} \geq 0
\end{gathered}
$$

(the derivatives are meant with respect to $r$ ). These conditions can be rewritten in terms of the function $\psi(y)$ introduced above. More precisely, since $f(r)=$ $\left.\Phi(t)\right|_{t=\log r}, y=\Phi^{\prime}(t), \psi(y)=\Phi^{\prime \prime}(t)$, we have $f^{\prime}=\frac{y}{r}, f^{\prime \prime}=\frac{\psi-y}{r^{2}}, f^{\prime \prime \prime}=\frac{\psi^{\prime} \psi-3 \psi+2 y}{r^{3}}$ and (46) rewrite 


$$
\begin{gathered}
y \geq 0 \\
\psi-y+y^{2} \geq 0 \\
3 y \psi+\psi^{\prime} \psi-3 \psi+2 y-3 y^{2}+y^{3} \geq 0
\end{gathered}
$$

Now, by replacing $\psi(y)=A y^{2}+y+B$ in (47) one gets

$$
\begin{gathered}
y \geq 0 \\
(A+1) y^{2}+B \geq 0 \\
(2 A y+3 y-2)\left(A y^{2}+y+B\right)+2 y-3 y^{2}+y^{3} \geq 0
\end{gathered}
$$

From the second and the third condition one immediately deduces that if the metric is such that in the interval of definition one can let $y=\Phi^{\prime}(t)$ tend to zero, then it must be $B=0$, as claimed.

Lemma 3.4. Let $g$ be a radial Kähler metric as above and let $\psi(y)=A y^{2}+y+B$ given by (30). Assume $g$ is projectively induced and $y_{0}$ is a limit point in the domain of $\psi$. If $y_{0}$ is a positive root of $\psi$ then $y_{0} \in \mathbb{Z}$.

Proof. For every $k \geq 1$ one can prove by induction on $k$ that

$$
e^{-f} \frac{d^{k} e^{f}}{d r^{k}}=\frac{\psi P_{k}+y(y-1)(y-2) \cdots(y-k+1)}{r^{k}},
$$

where $P_{k}$ is a polynomial in $y$. Indeed, for $k=1$ one has

$$
e^{-f} \frac{d e^{f}}{d r}=f^{\prime}=\frac{F^{\prime}(t)}{r}=\frac{y}{r}
$$

that is (49) with $P_{0}=0$. Now, assuming by induction that (49) is true for some $k$, we claim that it is true for $k+1$. Indeed, one has

$$
\begin{aligned}
\frac{d^{k+1} e^{f}}{d r^{k+1}} & =\frac{d}{d y}\left[\psi P_{k}+y(y-1)(y-2) \cdots(y-k+1)\right] \frac{d y}{d r} \cdot \frac{e^{f}}{r^{k}}+ \\
& +\left[\psi P_{k}+y(y-1)(y-2) \cdots(y-k+1)\right] \frac{d}{d r}\left(\frac{e^{f}}{r^{k}}\right)
\end{aligned}
$$

and the claim follows by using

$$
\begin{gathered}
\frac{d y}{d r}=\frac{d}{d r} F^{\prime}(t)=\frac{F^{\prime \prime}(t)}{r}=\frac{\psi}{r} \\
\frac{d}{d r}\left(\frac{e^{f}}{r^{k}}\right)=\frac{r f^{\prime}-k}{r^{k+1}} e^{f}=\frac{y-k}{r^{k+1}} e^{f}
\end{gathered}
$$

and the fact that $\psi$ is a polynomial. Now, notice that from (49) it follows that if $y_{0}$ is a root of $\psi$, then

$$
\left.e^{-f} \frac{d^{k} e^{f}}{d r^{k}}\right|_{y=y_{0}}=\frac{y_{0}\left(y_{0}-1\right)\left(y_{0}-2\right) \cdots\left(y_{0}-k+1\right)}{r^{k}}
$$

Assume $y_{0} \notin \mathbb{Z}$. By continuity, in a neighbourhood of $y=y_{0}$ one has that $\frac{d^{k} e^{f}}{d r^{k}}$ has the same sign of $y_{0}\left(y_{0}-1\right)\left(y_{0}-2\right) \cdots\left(y_{0}-k+1\right)$ which is strictly negative for 
$k=\left[y_{0}\right]+2\left(\right.$ where $\left[y_{0}\right]$ denotes the integer part of $\left.y_{0}\right)$. Hence, by Lemma 3.2, the metric is not projectively induced.

Proof of Proposition 3.1. By the discussion before the statement of the proposition, we are left to prove that the metrics corresponding to cases (33), (36), (37), (39), (40) and (41) are not projectively induced for any values of $\lambda, \xi, \mu$, and that if $\lambda \notin \mathbb{Z}$ or $\frac{\lambda \mu}{2} \notin \mathbb{Z}$ then the metric (38) is not projectively induced.

By Lemma 3.3 one immediately sees that the metrics (33), (36), (37), (39), and (41) are not projectively induced for any values of the parameters.

For the remaining cases (38) and (40), we cannot use the same argument since we have respectively $\frac{\mu \lambda}{2}<y<+\infty$ and $\frac{\mu(1-\zeta)}{2}<y<\frac{\mu(1+\zeta)}{2}$ and so 0 is not a limit point in the domain of definition of $\psi$, so we will use another approach. More precisely, we will take the explicit expressions of the potentials of these metrics, which are respectively (see the statement of Theorem 2.1 in [23])

$$
f(r)=\log \frac{r^{\frac{\mu \lambda}{2}}}{\left(1-\xi r^{\lambda+1}\right)^{\mu}}
$$

and

$$
f(r)=\log \left[r^{\frac{\mu(1+\zeta)}{2}}\left(1+r^{-\zeta}\right)^{\mu}\right]
$$

and we will apply the criterion given in Lemma 3.2

Let us begin from case (40): by Lemma 3.4. if one of the two roots

$$
k=\frac{\mu(1-\zeta)}{2}, \quad l=\frac{\mu(1+\zeta)}{2}
$$

of $\psi$ is not an integer, then the metric is not projectively induced. Assume thus that $k, l \in \mathbb{Z}^{+}$, which implies also $k+l=\mu \in \mathbb{Z}$. Then, by (52) we have

$$
e^{f(r)}=r^{l}\left(1+r^{-\zeta}\right)^{\mu}=\sum_{s=0}^{\mu}\left(\begin{array}{l}
\mu \\
s
\end{array}\right) r^{l-\zeta s}=\sum_{s=0}^{\mu}\left(\begin{array}{c}
\mu \\
s
\end{array}\right) r^{k+(\mu-s) \zeta} .
$$

By a straight calculation one sees that, for $k_{0}=k+2$ one has

$$
\frac{d^{k_{0}}}{d r^{k_{0}}} e^{f(r)}=r^{\zeta-2}\left(c_{0}+c_{1} r^{\zeta}+c_{2} r^{2 \zeta}+\cdots+c_{\mu-1} r^{(\mu-1) \zeta}\right),
$$

for suitable constants $c_{j}$, with

$$
c_{0}=\mu(\zeta+k) \cdots(\zeta+1) \zeta(\zeta-1)
$$

is negative since $0<\zeta<1$. This implies that $\frac{d^{k} 0}{d r^{k} 0} e^{f(r)} \rightarrow-\infty$ for $r \rightarrow 0^{+}$and proves that the metric is not projectively induced for any values of the parameters.

For the last case (38), we first notice that, by Lemma 3.4 if the root $\frac{\mu \lambda}{2}$ of $\psi$ is not an integer then the metric is not projectively induced. We are then left to 
show that when $\frac{\mu \lambda}{2} \in \mathbb{Z}$ and $\lambda \notin \mathbb{Z}$ then the metric is not projectively induced. By (51) one has

$$
e^{f(r)}=\sum_{i=0}^{\infty} \frac{\mu(\mu+1) \cdots(\mu+i-1)}{i !} \xi^{i} r^{(\lambda+1) i+\frac{\mu \lambda}{2}}
$$

Then, by a straightforward computation, one sees that, for $k_{0}=\frac{\mu \lambda}{2}+[\lambda]+3$ (where $[\lambda]$ denotes the integer part of $\lambda$ )

$$
\frac{d^{k_{0}}}{d r^{k_{0}}} e^{f(r)}=r^{\lambda-[\lambda]-2}\left(c_{0}+c_{1} r^{\lambda+1}+c_{2} r^{2(\lambda+1)}+\cdots\right)
$$

where

$$
c_{0}=\mu \xi\left(\lambda+\frac{\mu \lambda}{2}+1\right)\left(\lambda+\frac{\mu \lambda}{2}\right) \cdots(\lambda-[\lambda])(\lambda-[\lambda]-1)
$$

is strictly negative since we are assuming $\lambda \notin \mathbb{Z}$. This implies that $\frac{d^{k_{0}}}{d r^{k_{0}}} e^{f(r)} \rightarrow-\infty$ for $r \rightarrow 0^{+}$and concludes the proof.

\section{The proofs of Theorem 1.2 And Theorem 1.3}

Proof of Theorem [1.2. By Theorem 1.1] the finiteness of TYCZ expansion implies that the Kempf distortion function reduces to the polynomial $T_{m g}=m+a_{1}$. This forces $a_{2}=0$ and hence, using again the fact that $M$ is a complex curve, one deduces by (5) that $a_{1}=\frac{1}{2} \mathrm{scal}_{g}=$ const, namely the metric $g$ is a cscK metric. Notice that, by completeness, if $(M, g)$ were simply-connected then one would deduce that it is a one-dimensional complex space form (a), (b) and (c), where $\lambda$ is a positive integer (we are also using the integrality of the Kähler form $\omega$ associated to $g$ to obtain the integrality of $\lambda$ ). Hence, in order to prove the theorem, we are reduced to show that $M$ is simply-connected. Assume, by contradiction, that $M$ is not simply connected and let $p:(\tilde{M}, \tilde{g}) \rightarrow(M, g)$ be the universal covering map (which is a non-injective Kähler immersion satisfying $\left.p^{*} g=\tilde{g}\right)$. Then $(\tilde{M}, \tilde{g})$ would be one of the three onedimensional complex space forms (a), (b), (c), and hence there exists an injective full Kähler immersion $\psi: \tilde{M} \rightarrow \mathbb{C P}^{N}$ (see Remark [ above). Since $T_{g}=1+a_{1}$ is constant one deduce (see (10)) that the coherent states map $\varphi_{1}: M \rightarrow \mathbb{C P}^{N(1)}$ is a full Kähler immersion. Hence the holomorphic map $\varphi_{1} \circ p: \tilde{M} \rightarrow \mathbb{C P}^{N(1)}$ satisfies $\left(\varphi_{1} \circ p\right)^{*} g_{F S}=p^{*} \varphi_{1}^{*} g_{F S}=\tilde{g}$. By the celebrated Calabi's rigidity theorem [6] $N(1)=N$ and there exists a unitary transformation $U$ of $\mathbb{C P}^{N}$ such that $U \circ \psi=\varphi_{1} \circ p$. This forces $\varphi_{1} \circ p$ and hence $p$ to be injective, yelding the desired contradiction.

Finally, we prove Theorem 1.3 
Proof of Theorem 1.3. Combining the assumptions with Theorem 1.1 and Remark 5 one gets that, for some constant $a_{1}$, the Kempf distortion function associated to $(M, g)$. is given by $T_{m g}=m^{2}+a_{1} m$. Therefore the metric $g$ is forced to be balanced for all $m$ (or equivalently $(L, h)$ is a regular quantization). Recall that a balanced metric is automatically projectively induced and, as we have already pointed out in the Introduction, (i), (ii), (iii) and (iv) in Theorem 1.3 all admit an open and dense subset with a cscK metric with radial potential with finite TYCZ expansion. Thus, by using Proposition 3.1 we are left to show that the metric of case (38) of the classification in Section 3, given by potential (42), does not admit a regular quantization for $\xi, \lambda, \mu>0$ with $\lambda, \frac{\lambda \mu}{2} \in \mathbb{Z}$. In order to do that, recall that by (10) this happens if and only if

$$
\frac{i}{2 \pi} \partial \bar{\partial} \log \sum_{j}\left|s_{j}(z)\right|^{2}=\frac{i}{2 \pi} \partial \bar{\partial} \hat{\Phi}
$$

where $\left\{s_{j}\right\}$ is an orthonormal basis of the space $\mathcal{H}_{\mu, \lambda, \xi}$ of holomorphic functions $s=s(z)$ on the domain of definition of the metric

$$
U=\left\{r=\left|z_{1}\right|^{2}+\left|z_{2}\right|^{2} \mid r<\left(\frac{1}{\xi}\right)^{\frac{1}{\lambda+1}}\right\}
$$

which are bounded with respect to the norm

$$
\|s\|_{h_{\mu}}^{2}=\int_{U} h_{\mu}(z)|s(z)|^{2} d v(z)
$$

endowed with the hermitian product $6\langle s, t\rangle_{h_{\mu}}=\int_{U} h_{\mu}(z) s(z) \overline{t(z)} d v(z)$ (cf. (11) and (12) in the introduction), where

$$
h_{\mu}(z)=e^{-\hat{\Phi}(z)}=\frac{\left[1-\xi r^{(\lambda+1)}\right]^{\mu}}{r^{\frac{\mu \lambda}{2}}}, \quad r=\left|z_{1}\right|^{2}+\left|z_{2}\right|^{2}
$$

and $d v(z)=\left(\frac{i}{2 \pi}\right)^{2} \operatorname{det}(g) d z_{1} \wedge d \bar{z}_{1} \wedge d z_{2} \wedge d \bar{z}_{2}$ is the volume form.

Now, take $s(z)=z_{1}^{j} z_{2}^{k}$. By passing to polar coordinates $z_{1}=\rho_{1} e^{i \theta_{1}}, z_{2}=\rho_{2} e^{i \theta_{2}}$, and using (44) we get

$$
\begin{aligned}
\|s\|_{h_{\mu}}^{2} & =\int_{U}\left|z_{1}\right|^{2 j}\left|z_{2}\right|^{2 k} h_{\mu} d v(z) \\
& =2 \xi \mu^{2}(\lambda+1)^{2} \int \rho_{1}^{2 j+1} \rho_{2}^{2 k+1} \frac{\left(1-\xi\left(\rho_{1}^{2}+\rho_{2}^{2}\right)^{\lambda+1}\right)^{\mu}}{\left(\rho_{1}^{2}+\rho_{2}^{2}\right)^{\frac{\mu \lambda}{2}}} \frac{\left(\rho_{1}^{2}+\rho_{2}^{2}\right)^{\lambda-1}\left[\lambda+\xi(\lambda+2)\left(\rho_{1}^{2}+\rho_{2}^{2}\right)^{\lambda+1}\right]}{\left(1-\xi\left(\rho_{1}^{2}+\rho_{2}^{2}\right)^{\lambda+1}\right)^{3}} d \rho_{1} d \rho_{2},
\end{aligned}
$$

where we are integrating on $\rho_{1}^{2}+\rho_{2}^{2}<\left(\frac{1}{\xi}\right)^{\frac{1}{\lambda+1}}$.

\footnotetext{
${ }^{6}$ Notice that we are using the fact that $U$ is dense in $M$ in order to integrate on $U$.
} 
Now by setting $\rho=\sqrt{\rho_{1}^{2}+\rho_{2}^{2}}$ we can make the substitution $\rho_{1}=\rho \cos \theta, \rho_{2}=$ $\rho \sin \theta, 0<\rho<\infty, 0<\theta<\frac{\pi}{2}$, and using

$$
\int_{0}^{\frac{\pi}{2}}(\cos \theta)^{2 j+1}(\sin \theta)^{2 k+1}=\frac{j ! k !}{2(j+k+1) !}
$$

the previous integral becomes

$$
\xi \mu^{2}(\lambda+1)^{2} \frac{j ! k !}{(j+k+1) !} \int_{0}^{\left(\frac{1}{\xi}\right)^{\frac{1}{2(\lambda+1)}}} \rho^{2 j+2 k+2 \lambda-\mu \lambda+1} \frac{\lambda+\xi(\lambda+2) \rho^{2(\lambda+1)}}{\left(1-\xi \rho^{2(\lambda+1)}\right)^{3-\mu}} d \rho .
$$

Let us make the change of variable

$$
x=\xi \rho^{2(\lambda+1)}, \quad d x=2 \xi(\lambda+1) \rho^{2 \lambda+1} d \rho
$$

and (55) rewrites

$$
\xi^{-\frac{2 j+2 k-\mu \lambda}{2(\lambda+1)}} \mu^{2}(\lambda+1) \frac{j ! k !}{(j+k+1) !} \int_{0}^{1} x^{\frac{2 j+2 k-\mu \lambda}{2(\lambda+1)}} \frac{\lambda+(\lambda+2) x}{(1-x)^{3-\mu}} d x
$$

and then one easily sees that it converges if and only if $\mu>2$ and $\frac{2 j+2 k-\mu \lambda}{2(\lambda+1)}>-1$, i.e.

$$
j+k>\frac{\mu \lambda}{2}-(\lambda+1) .
$$

This is the condition for a monomial $z_{1}^{j} z_{2}^{k}$ to belong to the space $\mathcal{H}_{\mu, \lambda, \xi}$. Since by radiality it is easy to see that the monomials $z_{1}^{j} z_{2}^{k}$ are pairwise orthogonal, we see that $\left\{z_{1}^{j} z_{2}^{k}\right\}_{j+k>\frac{\mu \lambda}{2}-(\lambda+1)}$ form a complete orthogonal basis of in $\mathcal{H}_{\mu, \lambda, \xi}$, so the condition (53) for the metric to be balanced can be rewritten

$$
\frac{i}{2 \pi} \partial \bar{\partial} \log \left[\sum_{j+k>\frac{\mu \lambda}{2}-(\lambda+1)} \frac{\left|z_{1}\right|^{2 j}\left|z_{2}\right|^{2 k}}{\left\|z_{1}^{j} z_{2}^{k}\right\|_{h_{\mu}}^{2}}\right]=\frac{i}{2 \pi} \partial \bar{\partial} \hat{\Phi}
$$

This means that there exists a holomorphic function $f$ such that

$$
\log \left[\sum_{j+k>\frac{\mu \lambda}{2}-(\lambda+1)} \frac{\left|z_{1}\right|^{2 j}\left|z_{2}\right|^{2 k}}{\left\|z_{1}^{j} z_{2}^{k}\right\|_{h_{\mu}}^{2}}\right]=\hat{\Phi}+\operatorname{Re}(f) .
$$

By radiality, $f$ is forced to be constant and we can rewrite this condition as

$$
\sum_{j+k>\frac{\mu \lambda}{2}-(\lambda+1)} \frac{\left|z_{1}\right|^{2 j}\left|z_{2}\right|^{2 k}}{\left\|z_{1}^{j} z_{2}^{k}\right\|_{h_{\mu}}^{2}}=C e^{\hat{\Phi}}=C \frac{\left(\left|z_{1}\right|^{2}+\left|z_{2}\right|^{2}\right)^{\frac{\mu \lambda}{2}}}{\left[1-\xi\left(\left|z_{1}\right|^{2}+\left|z_{2}\right|^{2}\right)^{(\lambda+1)}\right]^{\mu}}
$$

for some $C>0$.

Now, we notice that since we are assuming $\lambda>0$, then condition (57) is fulfilled for $j+k=\frac{\mu \lambda}{2}-1$ (recall that $\lambda, \mu>0$ and that we are assuming that $\frac{\mu \lambda}{2} \in \mathbb{Z}$, otherwise the metric is not projectively induced). But it is easy to see that the 
Taylor expansion of the right-hand side of (59) does not contain the term $\left|z_{1}\right|^{2 j}\left|z_{2}\right|^{2 k}$ for $j+k=\frac{\mu \lambda}{2}-1$, so (59) cannot be satisfied and the metric is not balanced. This concludes the proof of the theorem.

\section{REFERENCES}

[1] C. Arezzo, A. Loi, Quantization of Kähler manifolds and the asymptotic expansion of TianYau-Zelditch, J. Geom. Phys. 47 (2003), 87-99.

[2] C. Arezzo and A. Loi, Moment maps, scalar curvature and quantization of Kähler manifolds, Comm. Math. Phys. 246 (2004), 543-549.

[3] C. Arezzo, A. Loi, F. Zuddas, On homothetic balanced metrics, Ann. Global Anal. Geom. 41, n. 4 (2012), 473-491.

[4] C. Arezzo, A. Loi, F. Zuddas, Szegö Kernel, regular quantizations and spherical CRstructures. Math. Z. (2013) 275, 1207-1216.

[5] L. Boutet de Monvel and J. Sjöstrand, Sur la singularité des noyaux de Bergman et de Szegö, Journes: Equations aux Dérivées Partielles de Rennes (1975), Soc. Math. France, Paris, 1976, pp. 123-164. Astèrisque, No. 3435.

[6] E. Calabi, Isometric Imbedding of Complex Manifolds, Ann. of Math. Vol. 58 No. 1, 1953.

[7] D. Catlin, The Bergman kernel and a theorem of Tian, In: Analysis and geometry in several complex variables (Katata, 1997), Trends Math., pp. 1-23, Boston, MA (1999).

[8] F. Cannas Aghedu, A. Loi, The Simanca metric admits a regular quantization, arXiv:1809.04431

[9] M. Cahen, S. Gutt, J. H. Rawnsley, Quantization of Kähler manifolds I: Geometric interpretation of Berezin's quantization, JGP. 7 (1990), 45-62.

[10] D. Catlin, The Bergman kernel and a theorem of Tian, in Analysis and geometry in several complex variables (Katata, 1997), Trends Math., Birkhuser Boston, Boston, MA (1999), 1-23.

[11] S. Donaldson, Scalar Curvature and Projective Embeddings, I, J. Diff. Geometry 59 (2001), 479-522.

[12] M. Engliš, Berezin Quantization and Reproducing Kernels on Complex Domains, Trans. Amer. Math. Soc. vol. 348 (1996), 411-479.

[13] M. Engliš, A Forelli-Rudin construction and asymptotics of weighted Bergman kernels, J. Funct. Anal. 177 (2000), no. 2, 257-281.

[14] C. Fefferman, The Bergman kernel and biholomorphic mappings of pseudoconvex domains, Invent. Math. 26 (1974), 1-65.

[15] T. Gramchev, A. Loi, TYZ expansion for the Kepler manifold, Comm. Math. Phys. 289, (2009), 825-840.

[16] G. R. Kempf, Metric on invertible sheaves on abelian varieties, Topics in algebraic geometry (Guanajuato) (1989).

[17] S. Ji, Inequality for distortion function of invertible sheaves on Abelian varieties, Duke Math. J. 58 (1989), 657-667.

[18] A. Loi, The Tian-Yau-Zelditch asymptotic expansion for real analytic Kähler metrics, Int. J. of Geom. Methods Mod. Phys. 1 (2004), 253-263.

[19] A. Loi, A Laplace integral, the T-Y-Z expansion and Berezin's transform on a Kähler manifold, Int. J. Geom. Meth. in Mod. Ph. 2 (2005), 359-371.

[20] A. Loi, R. Mossa, Berezin quantization of homogeneous bounded domains, Geom. Dedicata 161 (2012), 119-128. 
[21] A. Loi, R. Mossa, F. Zuddas, The log-term of the disc bundle over a homogeneous Hodge manifold, Ann. Global Anal. Geom. 51 (2017), no. 1, 35-51.

[22] A. Loi, F. Salis, F. Zuddas, Two conjectures on Ricci flat metrics, Math. Z. 290, 599-613 (2018).

[23] A. Loi, F. Salis, F. Zuddas, On the third coefficient of TYZ expansion for radial scalar flat metrics, J. Geom. Phys. 133, 210-218 (2018).

[24] A. Loi, M. Zedda, F. Zuddas Some remarks on the Kähler geometry of the Taub-NUT metrics, Ann. of Glob. Anal. and Geom., Vol. 41 n.4 (2012), 515-533.

[25] A. Loi, M. Zedda, On the coefficients of TYZ expansion of locally Hermitian symmetric spaces, Manuscripta Mathematica (2015),Vol. 148, 303-315.

[26] A. Loi, M. Zedda, Kähler Immersions of Kähler Manifolds into Complex Space Forms, Lecture Notes of the Unione Matematica Italiana 23, Springer, (2018).

[27] Z. Lu, On the lower order terms of the asymptotic expansion of Tian-Yau-Zelditch, Amer. J. Math. 122 (2000), no. 2, 235-273.

[28] Z. Lu and G. Tian, The log term of Szegö Kernel, Duke Math. J. 125 (2004), 351-387.

[29] X. Ma, G. Marinescu, Holomorphic morse inequalities and Bergman kernels, Progress in Mathematics, Birkhäuser, Basel, (2007).

[30] I. P. Ramadanov, A characterization of the balls in $\mathbb{C}^{n}$ by means of the Bergman kernel, C. R. Acad. Bulgare Sci. 34 (1981), 927-929.

[31] J. Rawnsley, Coherent states and Kähler manifolds, Quart. J. Math. Oxford (2), n. 28 (1977), 403-415.

[32] W. D. Ruan, Canonical coordinates and Bergmann metrics, Comm. in Anal. and Geom. (1998), 589-631.

[33] G. Tian, On a set of polarized Kähler metrics on algebraic manifolds, J. Diff. Geometry 32 (1990), 99-130.

[34] S. Zhang, Heights and reductions of semi-stable varieties, Comp. Math. 104 (1996), 77-105.

[35] S. Zelditch, Szegö kernels and a theorem of Tian Int. Math. Res. Notices 6, 317-331 (1998).

(Andrea Loi) Dipartimento di Matematica, Università di Cagliari (Italy)

E-mail address: loi@unica.it

(Roberto Mossa) Departamento de Matemática, Universidade Federal de Santa CataRINA (BRASIL)

E-mail address: roberto.mossa@gmail.com

(Fabio Zuddas) Dipartimento di Matematica e Informatica, Università di Cagliari (ITALY)

E-mail address: fabio.zuddas@unica.it 